\title{
Combining Edge, Region, and Shape Information to Segment the Left Ventricle in Cardiac MR Images
}

\author{
Marie-Pierre Jolly \\ Imaging and Visualization Department \\ Siemens Corporate Research \\ Princeton, NJ \\ jolly@scr.siemens.com
}

\begin{abstract}
This paper describes a segmentation technique to automatically extract the myocardium in 4D cardiac MR images for quantitative cardiac analysis and the diagnosis of patients. An approximate outline of the left ventricle is obtained either from automatic localization based on the maximum discrimination method or from copying a template shape during propagation. The histogram of the image is analyzed and divided into peaks using the EM algorithm to produce a region-based segmentation. This result and the image gradient are combined to obtain candidate boundaries for the left ventricle by deforming the contour using a graph search active contour approach. The final boundary is chosen using a minimum cut graph algorithm, spline fitting, or point pattern matching to maintain the shape of the template. We have experimented with the proposed method on a large number of patients and present some quantitative and qualitative results.
\end{abstract}

\section{Introduction}

Cardiovascular disease is the leading cause of death in the United States. Mortality has been declining over the years as lifestyle has changed, but the decline is also due to the development of new technologies to diagnose disease. One of these techniques is magnetic resonance imaging (MRI) which provides time-varying three-dimensional imagery of the heart. To help in the diagnosis of disease, the physicians are interested in identifying the heart chambers, the endocardium and epicardium, and measuring the change in ventricular blood volume (ejection fraction) and wall thickening properties over the cardiac cycle. The left ventricle is of particular interest since it pumps oxygenated blood out to distant tissue in the entire body.

There has been a large amount of research on the analysis of medical images [1] and the segmentation problem has been particularly challenging. In the early nineties, researchers have realized that tracking the cardiac wall motion in MR images was important to characterize meaningful functional changes. The system proposed by Fleagle et al. [2] was able to delineate the borders of the myocardium using a minimum cost path graph search algorithm after the user indicated the center of the left ventricular cavity and the area of interest with a few mouse clicks. Geiger et al. [3] used a dynamic programming approach to refine the contours specified by the user. Goshtasby and Turner [4] proposed a two step algorithm combining intensity 
thresholding to recover the bright blood and local gradient to outline the strong edges using elastic curves. Weng et al. [5] thresholded the image based on parameters estimated during a learning phase to get a good approximate of the segmentation. Although we have not directly used any of these techniques, all of these papers have greatly influenced our work.

Argus is a cardiac analysis package developed by Siemens which offers a complete system of drawing tools and automatic segmentation algorithms to allow the physician to outline the myocardium in each image in the patient data set, compute volumes, ejection fraction, and perform a thickening analysis. In this paper, we present the segmentation module which combines edge, region, and shape information in a deformable template approach.

\section{Approximate Localization of the Left Ventricle}

As for any deformable template based algorithm, our method requires an approximate delineation of the object of interest to be provided. We have developed an algorithm to automatically hypothesize two concentric circles at the location of the left ventricle in a new image. We use a method similar to the maximum discrimination method proposed by Colmenarez and Huang [6]. However, due to the relative symmetry of the left ventricle and computational constraints, we have only used the gray values of the pixels along the main four cross sections through the ventricle instead of the entire region to derive the feature set. More details are given in [7] and [8].

The system models patterns as a Markov process. In the learning phase, positive and negative examples are presented and the system finds the ordering of the Markov process that maximizes the separation (minimizes the Kullbach distance) between the two classes in the training set. In the detection stage, the test image is scanned and each location is assigned to the closest class based on the log-likelihood ratio.

Neighboring positions classified as left ventricle are partitioned into clusters. We define 8 salient points in the gray level profiles as the intersection of the 4 cross sections with the ventricle's medial axis. Average profiles were built from training example profiles which were aligned using the curve registration technique proposed by Ramsay and Li [9]. The cross sections of each of the cluster candidates are warped onto their corresponding average profiles. The location of the salient points in the image are then accumulated using a Hough transform array to vote for the most likely center position and radius for the myocardium centerline.

Typically, the user segments one image, usually the slice closest to the valves at end-diastole (ED base) and propagates the segmented contours to all the slices in the ED phase (ED propagation). Then, all the ED contours are propagated to the endsystole (ES) phase (ES propagation) to compute the ejection fraction. For a more detailed analysis, the user can also propagate all the ED contours to all the images in all the phases using temporal propagation.

For ED propagation, we use the automatic localization algorithm just described, but since the ventricle size is approximately known from the template image, we limit the scale search to 0.85 to 1.15 times the size of the template. We also limit the search space for the location of the ventricle to 30 pixels around the location of the template, instead of the entire image. For ES propagation, we know that the location of the left 
ventricle has not changed, so we simply scale the template contours. The endocardium is scaled by 0.6 and the epicardium by 0.9 . In the case of temporal propagation, both the location and scale of the contours barely change. So, we simply copy the contours from one image to the next.

These contours, obtained either through automatic localization or propagation (see Fig. 1) are the starting point to our local deformation process.

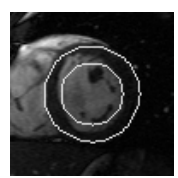

(a)

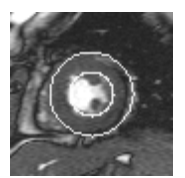

(b)

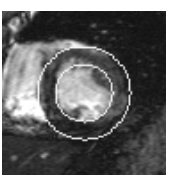

(c)

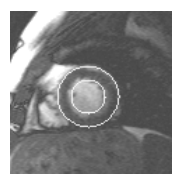

(d)

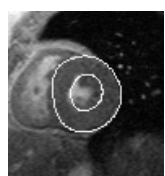

(e)

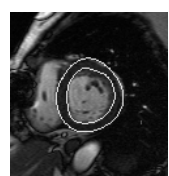

(f)

Fig. 1. Examples of approximate contours: (a)-(c) From automatic localization; (d) From ED propagation; (e) From ES propagation; (f) From temporal propagation.

\section{Local Deformations}

\subsection{Region Segmentation}

In MR imaging, the intensity of a pixel depends on the properties of the tissue being imaged. In our MR sequences of the left ventricle, the blood is very bright, the muscles are somewhat dark, but not as dark as the air-filled lungs. This fact can be verified by looking at the histogram of a region around the myocardium (see Fig. 2). We use the Expectation-Maximization (EM) algorithm [10] to fit a mixture of 3 Gaussians to the histogram. We then create a myocardium response image, showing the probability that a pixel belongs to the middle Gaussian which corresponds to the myocardium. It can be seen from Fig. 2(c) that the left ventricle myocardium is nicely highlighted, but neighboring organs are highlighted too.

\subsection{Active Contours}

To complement the results of region segmentation, we use an active contour formulation similar to Geiger et al.'s [3] dynamic programming approach or Mortensen and Barrett's [11] Dijkstra's approach. The advantage of these graph theoretic methods over the traditional gradient descent approach proposed by Kass et al. [12] is that they are able to recover the global optimum of the energy function and are therefore insensitive to the initial contour position.

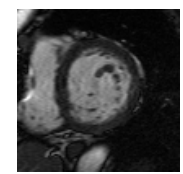

(a)

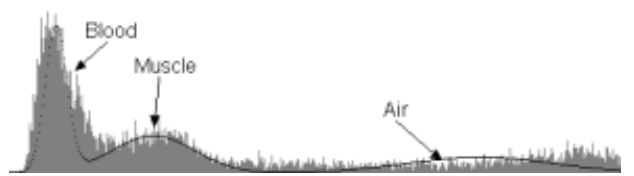

(b)

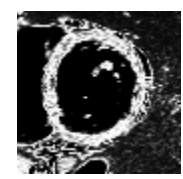

(c)

Fig. 2. Region segmentation and myocardium response image. 
Geiger et al. [3] define the energy of a contour $\left(p_{1}, \ldots p_{n}\right)$ as:

$$
E\left(p_{1}, \ldots, p_{n}\right)=\sum_{i=1}^{n} \frac{1}{\left\|\nabla I\left(p_{i}\right)\right\|+\varepsilon}+\alpha \sum_{i=2}^{n}\left|\vec{\nabla} I\left(p_{i}\right)-\vec{\nabla} I\left(p_{i-1}\right)\right|
$$

where $\|\nabla I(p)\|$ is the magnitude and $\vec{\nabla} I(p)$ the direction of the image gradient at pixel $p$. This is equivalent to finding the shortest path in a graph where nodes correspond to pixels and the cost of a link between two neighboring pixels is defined as:

$$
e\left(p_{1}, p_{2}\right)=\frac{1}{\left\|\nabla I\left(p_{2}\right)\right\|+\varepsilon}+\alpha\left|\vec{\nabla} I\left(p_{2}\right)-\vec{\nabla} I\left(p_{1}\right)\right|
$$

Given an approximate contour in an image as in Fig. 3(a), we place a symmetrical search space around it and define a line of source nodes (all connected to a "pseudo" source node) and sink nodes as in Fig. 3(b). Dijkstra's algorithm then finds the shortest path between the pseudo source node and one of the sink nodes as seen in Fig. 3(c). Unfortunately, there is no guarantee that this contour will be closed. So we define a new single source point in the middle of the recovered contour as in Fig. 3(d) and do a second pass of Dijkstra's algorithm to produce the final closed contour as in Fig. 3(e).

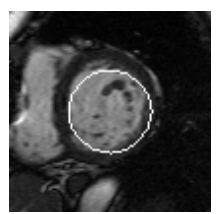

(a)

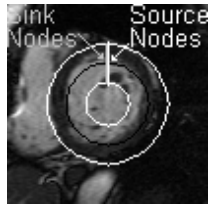

(b)

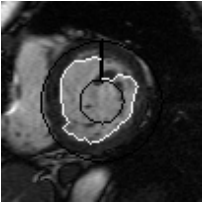

(c)

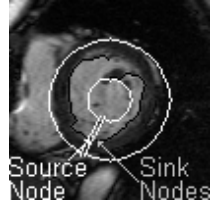

(d)

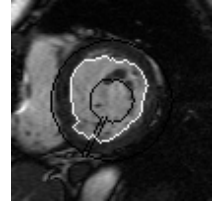

(e)

Fig. 3. Two passes of Dijkstra's algorithm are used to recover a closed contour.

\subsection{Energy Function}

To combine the information provided by both the image and the myocardium response image, we have chosen to run Dijkstra's algorithm with two different energy functions. Each run gives different candidate points for the contours, along with a confidence value based on the contribution of that point to the total energy function. One energy function combines gradient magnitude and direction using the cross product of the gradient direction and the contour direction. In our case, the contour is built clockwise by the Dijkstra process and the image gradient points from bright to dark. To separate a bright region inside from a dark region outside (resp. a dark region inside from a bright region outside), the $z$ component of the cross product between the image gradient and the contour direction should be positive (resp. negative). Thus, we set the energy to a large number otherwise. The cost of a link between two pixels is:

$$
e\left(I, z>0, p_{1}, p_{2}\right)=\left\{\begin{array}{cc}
\frac{1}{\left\|\nabla I\left(p_{2}\right)\right\|^{2}+\varepsilon} & \text { if } z=\begin{array}{c}
\left(x_{2}-x_{1}\right) \sin \left(\vec{\nabla} I\left(p_{2}\right)\right)- \\
1 / \varepsilon
\end{array} \\
\begin{array}{c}
\left(y_{2}-y_{1}\right) \cos \left(\vec{\nabla} I\left(p_{2}\right)\right)>0 \\
\text { otherwise }
\end{array}
\end{array}\right.
$$


where $\varepsilon$ is a small constant (0.001) to bound the energy function. We use $E(I, z>0)$ and $E(H, z<0)$ for the endocardium, where $I$ is the input image and $H$ is the myocardium response image. For the epicardium, we use $E(H, z>0)$. Since the gradient direction in $I$ outside the myocardium flips between the bright right ventricle and the dark lungs, we also use the energy function based on gradient magnitude only $E^{\prime}(I)$ defined by the cost between two pixels:

$$
e^{\prime}\left(I, p_{1}, p_{2}\right)=\frac{1}{\left\|\nabla I\left(p_{2}\right)\right\|^{2}+\varepsilon}
$$

In Fig. 4 it can be seen that different energy functions highlight different features of the myocardium. In particular, $E(I, z>0)$ for the endocardium outlines the papillary muscles while $E(H, z<0)$ does not. Also, $E^{\prime}(I)$ for the epicardium outlines the fat while $E(H, z>0)$ outlines the true border of the myocardium.

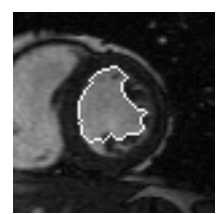

$E(I, z>0)$

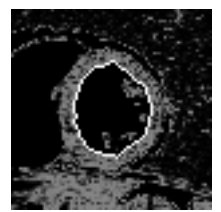

$E(H, z<0)$

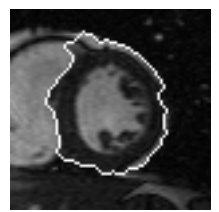

$E^{\prime}(I)$

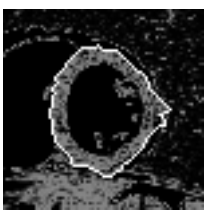

$E(H, z>0)$

Fig. 4. Various energy functions can be used to recover a contour using Dijkstra's algorithm.

\subsection{Shape Constraint}

Dijktra's algorithm is applied with various energy functions and all points on the resulting contours are candidate points for the final contour. When the contours are propagated from one image to the next, either spatially or temporally, the shape of the contours does not change drastically. Based on this assumption, we can decide which parts of which contours are correct.

We use the shape alignment method proposed by Duta et al. [13] to establish the correspondence between a subset $A^{\prime}$ of the template points $A=\left\{A_{j}\right\}_{j=1, \ldots, a}$ and a subset $B$ ' of the candidate test points $B=\left\{B_{k}\right\}_{k=1, \ldots, b}$. Best results are obtained when endocardium and epicardium are considered together as one shape. Given a pair of "corresponding" points in $A$ and $B$, we hypothesize a rigid similarity transform to align them. We then determine a one-to-one match matrix $M$ by assigning every point in $B$ to its closest neighbor in $A$ (if the distance is less than a threshold). This allows us to compute the distance:

$$
f(M)=\frac{1}{n^{2}} \sum_{j=1}^{n} w_{j}\left[\left(x_{A_{j}}-a x_{B_{j}}+c y_{B_{j}}-b\right)^{2}+\left(y_{A_{j}}-a y_{B_{j}}-c x_{B_{j}}-d\right)^{2}\right]+\frac{2}{n}
$$

where $n$ is the number of correspondences. We set the weight $w_{j}$ to be the confidence value of test point $B_{j}$ which is the inverse of the contribution of this point to the entire contour energy. The goal is to find the coefficients $(a, b, c, d)$ of the similarity transform which minimize $f(M)$. Of course, it is not possible to evaluate all possible 
quadruplets of points, so we choose the $10 \%$ of the points with largest confidence in the test set and pair them with points from the same contour in the template set.

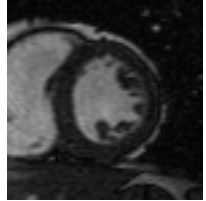

(a)

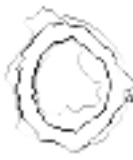

(b)

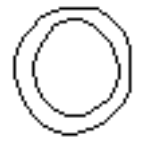

(c)

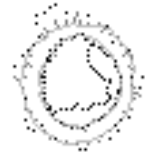

(d)

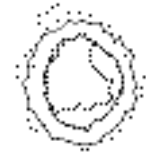

(e)

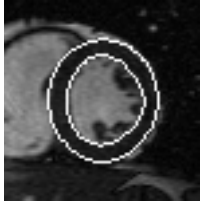

(f)

Fig. 5. Applying shape constraints to recover the myocardium contours.

Fig. 5(a) shows the input image and Fig. 5(b) the 4 recovered contours where darker points show higher confidence. Fig. 5(c) shows the template shape being propagated. Fig. 5(d) shows the established correspondences for the best similarity transform. The shape constraints allow the system to choose the outside candidate points for the endocardium (rather than the inside points that outline the papillary muscles) and the inside candidate points for the epicardium (rather than the outside points that outline the fat). Once the correspondences are established, the template shape is warped by moving the template points to their corresponding test points as in Fig. 5(e). Finally, the contours are smoothed using the method proposed by Xu et al. [14] that minimizes shrinkage. The final segmentation result is shown in Fig. 5(f).

\subsection{No Shape Constraint}

When an image needs to be segmented on its own (not in the context of propagation), there is no shape information available to the system. In this case, we use the techniques that were used in the previous version of our segmentation algorithm presented in [8]. The confidence values of every point on candidate contours are modified by:

$$
C_{\text {endo }}(p)=C(p)\left(\frac{d(p, \Omega)}{\max _{p} d(p, \Omega)}\right)^{3} \quad C_{\text {epi }}(p)=C(p)\left(\frac{d(p, \text { endo })}{\max _{p} d(p, \text { endo })}\right)^{3}
$$

so that endocardium points far away from the centroid $\Omega$ are emphasized and epicardium points closer to endocardium points are also emphasized.

Then, for the endocardium, we want to find the cycle with maximum confidence. We define a graph where each node corresponds to connected component regions between confidence pixels. The weight of an edge between two nodes is inversely proportional to the confidence of pixels on the common boundary. Then, we seek the minimum cut between the center node and the outside node. For the epicardium, we mostly want the contour to be smooth since there is no clear edge between the myocardium and the liver and the right ventricle myocardium appears to merge into the left ventricle myocardium. So, we simply fit a spline through the points of the 2 candidate contours. More details on these techniques are given in [8]. 


\section{Results}

In order to test our algorithm, we have collected 29 patient data sets along with a manual segmentation of the ED and ES phases by radiologists, for a total of 458 segmented images. The images were acquired on Siemens MAGNETOM systems using two different pulse sequences. FLASH pulse sequences were traditionally used for MR cineangiography. Siemens recently pionneered the TrueFISP pulse sequences for cardiac cine imaging which present higher contrast-to-noise ratio without affecting temporal or spatial resolution. We collected 22 TrueFISP patients and 7 FLASH patients. Our database presents a great variety of heart shapes, image contrast, and edge crispness. The difficulty with FLASH images (Fig. 9(a) or (c)) is that the edges are very blurred. The challenge with TrueFISP images is that the papillary muscles are so well defined that it can be difficult to avoid outlining them. Most of the time, the user does not want to outline them and we will see that the shape constraint greatly helps in this task.

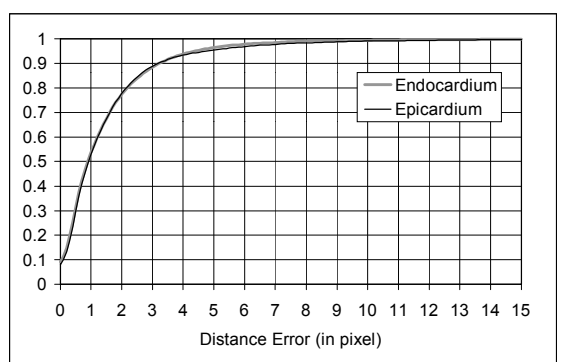

(a)

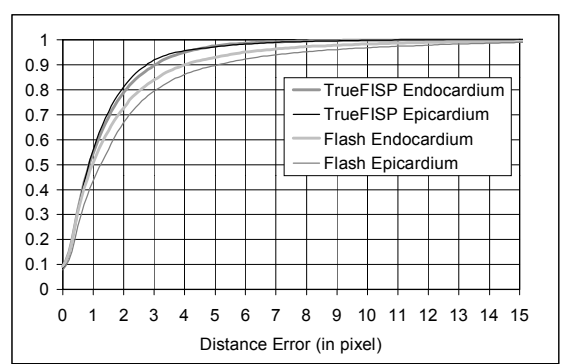

(b)

Fig. 6. Cumulative distribution of error distances between the true contours and the segmented contours, over all points, over all images, and over all patients.

We ran our algorithm to automatically segment the ED and ES phases of all the datasets. To compare the automatic contours $A$ with the true contours $B$, we compute the distances $d(a, B)=\min _{b \in B}\|a-b\|$ for all points $a$ in the automatic contour. Similarly, we compute $d(b, A)$ for all points $b$ in the true contour. Fig. 6(a) shows the cumulative distribution of these error distances over all ED and ES images of all 29 datasets. It can be seen that, on the average, the error is less than 1 pixel and an error of 5 pixels or more is made less than $5 \%$ of the time. Fig. $6(\mathrm{~b})$ shows the same curves separately for TrueFISP and FLASH data. It can be seen that the system performs better for TrueFISP data which is the standard acquisition mode on the new Siemens systems. The accurate localization of the epicardium on FLASH images is particularly difficult due to the weak edges. Nevertheless, in $90 \%$ of the cases, the error was less than 5 pixels.

Fig. 7 shows the segmentation of the whole heart, for all the slices in the ED phase. In a similar manner, Fig. 8 shows a temporal propagation for all the phases of a particular slice. Fig. 9(a) shows an example of ES propagation. It can be seen that the papillary muscles are kept inside the blood pool in the ES phase so that the shape of the endocardium is close to the shape defined in the ED phase. Fig. 9(b) shows another segmentation example. Fig. 9(c) shows a diseased heart where the cross 
section of the left ventricle appears elongated. Fig. 9(d) shows an example at the valve plane where the endocardium and the epicardium coincide. Fig. 9(e) shows an example where the myocardium is very thick and the papillary muscles very large. All these examples demonstrate the strength of our algorithm in handling different cases.

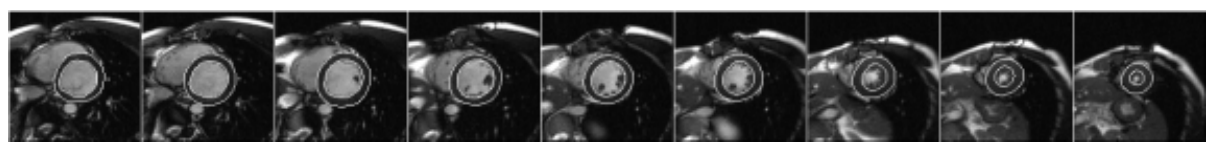

Fig. 7. Automatic localization followed by ED propagation for all the slices of the ED phase.

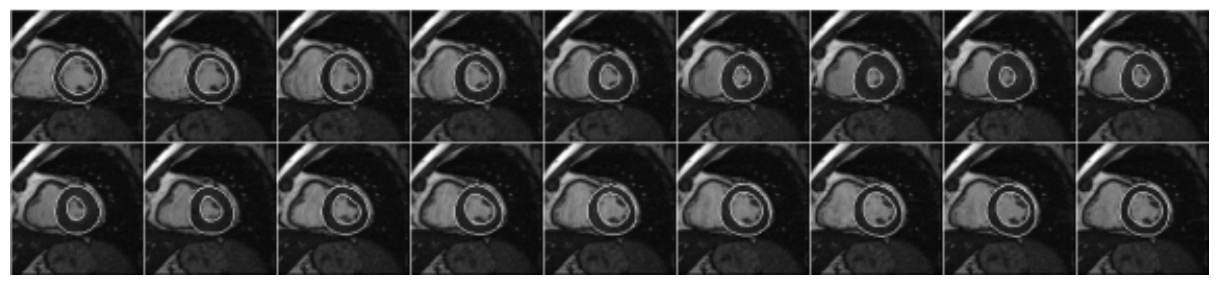

Fig. 8. Temporal propagation for all the phases of a particular slice.

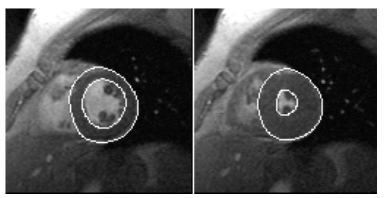

(a)

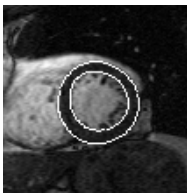

(b)

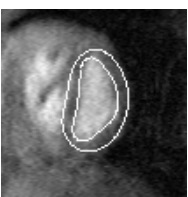

(c)

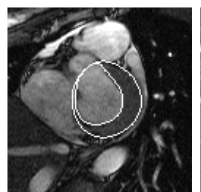

(d)

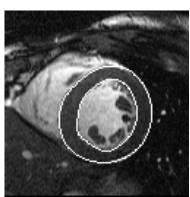

(e)

Fig. 9. ES propagation and other segmentation examples.

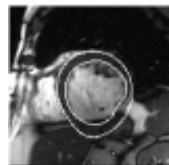

(a)

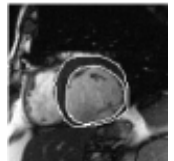

(b)

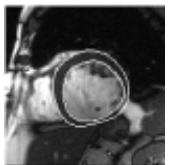

(c)

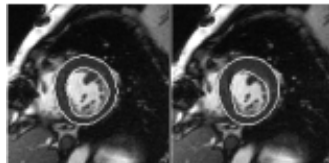

(d)

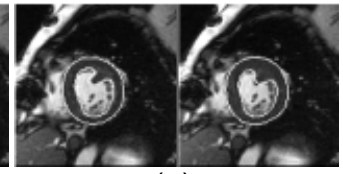

(e)

Fig. 10. Shape information is propagated to achieve the desired segmentation (see text).

Figure 10 illustrates the importance of the shape constraints. In Fig. 10(a) the left ventricle was automatically localized and local deformations were applied. Unfortunately, this patient has suffered an infarct in the past and the lower right side of the myocardium is very thin. In addition, there is no clear edge in the image between the myocardium and the liver. Thus, the resulting segmentation is wrong. Fig. 10(b) shows the previous slice in the volume where we have defined the desired contour manually. When the contour is propagated to the next slice, the shape constraint is enforced and the segmentation result is correct (as seen in Fig. 10(c)). Fig. 10(d) and (e) show temporal propagation. In Fig. 10(d) we chose to not outline the papillary muscle, whereas in Fig. 10(e), we decided to include the papillary muscle with the myocardium. In both cases, the contour propagated to the next image kept the shape outlined by the user. 


\section{Conclusions}

We have presented an algorithm to segment the left ventricle in cardiac MR images. The algorithm combines edge information, region information obtained from an EM fitting of a mixture of Gaussians to the histogram, and shape information through a point pattern matching strategy. We have obtained excellent results with this technique and integrated this algorithm with the next version of the cardiac analysis package Argus commercialized by Siemens.

\section{References}

1. J. S. Duncan and N. Ayache, "Medical image analysis: Progress over two decades and the challenges ahead", IEEE Trans. PAMI, 22(1):85-106, 2000.

2. S. R. Fleagle, D. R. Thedens, J. C. Ehrhardt, T. D. Scholz, and D. J. Skorton, "Automated identification of left ventricular borders from spin-echo resonance images", Investigative Radiology, 26:295-303, 1991.

3. D. Geiger, A. Gupta, L. A. Costa, and J. Vlontzos, "Dynamic programming for detecting, tracking, and matching deformable contours", IEEE Trans. PAMI, 17(3):294-302, 1995.

4. A. Goshtasby and D. A. Turner, "Segmentation of cardiac cine MR images for extraction of right and left ventricular chambers", IEEE Trans. Medical Imaging, 14(1):56-64, 1995.

5. J. Weng, A. Singh, and M. Y. Chiu, "Learning-based ventricle detection from cardiac MR and CT images", IEEE Trans. Medical Imaging, 16(4):378-391, 1997.

6. A. Colmenarez and T. Huang, "Face detection with information-based maximum discrimination”, Proc. IEEE CVPR, San Juan, Puerto Rico, pp 782-787, 1997.

7. N. Duta, A. K. Jain, and M.-P. Dubuisson-Jolly, "Learning-based object detection in cardiac MR images", Proc. ICCV, Corfu, Greece, pp 1210-1216, 1999.

8. M.-P. Jolly, N. Duta, and G. Funka-Lea, "Segmentation of the left ventricle in cardiac MR images", Proc. ICCV, Vancouver, Canada, 2001.

9. J. O. Ramsay and X. Li, "Curve registration", Journal of the Royal Statistical Society Series B, 60:351-363, 1998.

10. R. A. Redner and H. F. Walker, "Mixture densities, maximum likelihood and the EM algorithm", SIAM Review, 26:195-239, 1984.

11. E. N. Mortensen and W. A. Barrett, "Interactive segmentation with intelligent scissors", Graphical Models and Image Processing, 60:349-384, 1998.

12. M. Kass, A. Witkin, and D. Terzopoulos, "Snakes: Active contour models", International Journal of Computer Vision, 2:321-331, 1988.

13. N. Duta, A. K. Jain, M.-P. Dubuisson-Jolly, "Learning 2D shape models", Proc. IEEE CVPR, Fort Collins, CO, vol. II, pp. 8-14, 1999.

14. C. Xu, A. Yezzi Jr., J. L. Prince, "On the relationship between parametric and geometric active contours", Asilomar Conf. Signals, Systems, and Computers, pp. 483-489, 2000. 